\title{
Mapping the future for pulmonary fibrosis: report from the 17th International Colloquium on Lung and Airway Fibrosis
}

\author{
Luca Richeldi', Harold R. Collard², Roland M. du Bois ${ }^{3}$, Gisli Jenkins ${ }^{4}$, \\ Martin Kolb ${ }^{5}$, Toby M. Maher ${ }^{6}$, Ganesh Raghu ${ }^{7}$, Carlo Vancheri ${ }^{8}$ \\ and Geoffrey J. Laurent ${ }^{9,10}$
}

\begin{abstract}
Affiliations: ${ }^{1}$ Centre for Rare Lung Diseases, University of Modena and Reggio Emilia, Modena, and ${ }^{8}$ Dept of Internal Medicine, University of Catania, Catania, Italy. ${ }^{2}$ University of California, San Francisco, CA, and ${ }^{7}$ Division of Pulmonary and Critical Care Medicine, University of Washington, Seattle, WA, USA. ${ }^{3}$ Imperial College London, London, 'University of Nottingham, Nottingham, 'Interstitial Lung Disease Unit, Royal Brompton Hospital and National Heart and Lung Institute, Imperial College London, London, and ${ }^{10}$ University College London, London, UK. ${ }^{5}$ Dept of Respiratory Medicine, Pathology and Molecular Medicine, McMaster University, Hamilton, Canada. 'University of Western Australia, Perth, Australia. All authors were members of the scientific committee of the 17th ICLAF meeting.
\end{abstract}

Correspondence: L. Richeldi, Centre for Rare Lung Diseases, University Hospital of Modena, Via del Pozzo 71, 41124 Modena, Italy. E-mail: luca.richeldidunimore.it

ABSTRACT Pulmonary fibrosis is the ultimate outcome of various interstitial lung diseases, many of which have a dismal prognosis. Pulmonary fibrosis, therefore, represents a critical unmet medical need. Progress in research over the past 30 years has been encouraging. This work, which has been funded by governments, charitable trusts, industries and patient groups, has resulted in clinical trials testing novel drugs, giving hope to patients. In late September 2012, representatives from academia, industry and funding agencies met at the 17th International Colloquium on Airway and Lung Fibrosis to discuss state-of-the-art knowledge of pulmonary fibrosis. This manuscript summarises the outcomes of the meeting, highlighting the most relevant results and discoveries. It also attempts to provide a roadmap for future studies. It is hoped that such a roadmap may help interested parties to generate new research, which will be vital to continued progress. We are encouraged by the commitment expressed by all participants at this meeting and the shared vision of promoting future progress through international collaboration, the pooling of valuable resources, and the involvement of a new generation of physicians and scientists.

@ERSpublications

Progress report from the 17th international colloquium on lung and airway fibrosis: a roadmap for future studies http://ow.ly/kUltb

Received: March 042013 | Accepted after revision: April 172013 | First published online: May 162013

Conflict of interest: Disclosures can be found alongside the online version of this article at www.erj.ersjournals.com 
FIGURE 1 Key research steps in pulmonary fibrosis.

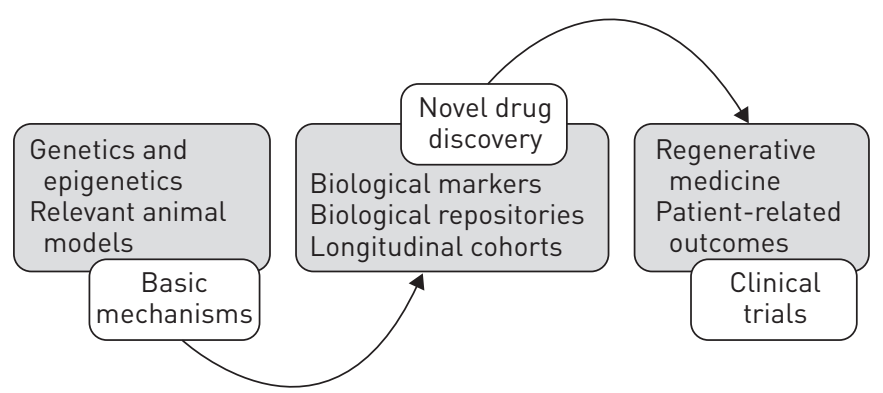

\section{ICLAF: a long journey}

The International Colloquium on Lung Fibrosis began in 1980, in London, UK, intended as a forum for intimate, in depth discussion of the basic and clinical science of fibrotic lung disease. With evolving knowledge of the pathogenesis of pulmonary fibrosis and the recognition that fibrosis within the lumen of distal airways is a component of some fibrotic lung diseases, the acronym ICLAF (International Colloquium on Lung and Airway Fibrosis) was coined, to include airway fibrosis, in 2008. The 17th ICLAF meeting took place between September 29 and October 3, 2012 (www.iclaf2012.unimore.it), when 258 participants from 22 different countries gathered together in Modena, Italy, for four days of presentations, lectures, posters and small group discussions. In attendance was a dynamic mix of clinicians, basic scientists, clinical researchers, industry colleagues and advocacy groups. This manuscript reviews the highlights of the conference and provides a "road map" for further research in this area, touching upon the main spheres of research in this rapidly evolving area of respiratory medicine (fig. 1). All sessions during the conference were video recorded and are freely available to everyone on a dedicated website [1].

\section{Invited lectures}

David Warburton (Los Angeles, CA, USA) placed fibrotic lung diseases in a developmental context, suggesting that abnormal development might contribute to the susceptibility to adult lung fibrosis and emphysema. Pulmonary emphysema and fibrosis may represent a useful model for the study of the potential synergistic effects of genetic defects in chronic lung disorders [2]. Microinjection of amniotic fluid stem cells (AFSC) into embryonic lungs showed that AFSC can differentiate into type II pneumocytes, while in the presence of an oxidative stress AFSC can integrate into the epithelium of the upper airways and alveoli. Moreover, transplantation of AFSC, which are safely collectable through amniocentesis samples without major ethical concerns, can retard the progression of bleomycin-induced lung fibrosis in the mouse via a type II cell dependent mechanism. These studies pave the way for a new approach using stem cells in pulmonary fibrosis.

Victor J. Thannickal (Birmingham, AL, USA) identified and attempted to reconcile some apparent paradoxes in our current understanding of the pathogenesis of idiopathic pulmonary fibrosis (IPF). Specifically, he discussed the "matrix paradox" and the "ageing paradox". Matrix stiffness transduces nuclear signalling events that regulate myofibroblast differentiation and survival via an intrinsic mechanotransduction pathway [3]. This mechanosensitive signalling pathway could be a target for anti-fibrotic therapies involving Rhoassociated protein kinase (ROCK) inhibitors such as fasudil, a molecule capable of inducing in vivo apoptosis of myofibroblasts following fibrogenic lung injury. Interestingly, inhibition of this mechanosensitive Rho kinase pathway with fasudil induces activation of the intrinsic mitochondrial apoptosis pathway in fibroblasts derived from patients with IPF, but not in fibroblasts from healthy donors. Furthermore, this approach ameliorates experimental pulmonary fibrosis [4]. Age-related diseases such as IPF may be associated with cellular senescence of specific lung cells. The data presented demonstrated that fibroblastic foci in IPF lungs express senescence markers; however, fibrosis appears to "paradoxically" require the proliferative expansion of fibroblasts. One oxidative stress-dependent pathway that leads to myofibroblast senescence and apoptosis resistance is NADPH oxidase 4 (NOX4). This might represent a promising therapeutic target in IPF [5] and potentially other chronic fibrotic disorders.

Seamas Donnelly (Dublin, Ireland) explored the complex interrelationships between viral infections and IPF. In particular, he presented new data supporting the hypothesis that repeated viral infection would predispose towards a more aggressive fibrotic response via a Toll-like receptor 3 (TLR3)-dependant process. The post hoc analysis of TLR3 polymorphisms in the cohort of a large, historical trial on the use of interferon (IFN) $-\gamma$ in IPF [6] showed that a single genetic polymorphism of the TLR3 gene is associated with accelerated lung function decline and enhanced mortality. These intriguing data prompted in vitro and animal studies, confirming the critical role of TLR3 and IFN in driving a more aggressive clinical phenotype in pulmonary fibrosis. This work highlights a significant genetic marker for enriching clinical trial 
populations with a more aggressive clinical phenotype. In addition, it potentially identifies specific therapies for this subgroup of IPF patients.

Kevin K. Brown (Denver, CO, USA) provided clinical insights into the development and progression of lung fibrosis. He described pulmonary fibrosis in multiple clinical contexts, highlighting infections, genetic conditions, systemic disorders and exposures. In each of these contexts a variety of pathological and radiographic patterns of pulmonary fibrosis can be seen. Regardless of context, the presence of clinically significant pulmonary fibrosis predicts the outcome, largely independent of the underlying cause of disease. For example, in connective tissue disease, survival is shortened in patients with rheumatoid arthritis. Unpublished data suggest that much of this effect can be attributed to the presence of interstitial lung disease (ILD) in these patients. The concept that progressive pulmonary fibrosis is the problem shared by a variety of clinical contexts has been clearly established. It has been the basis for a call to quickly address this unmet clinical need. The search for predictive biomarkers of progressive fibrosis is one of the key aims of a number of studies. In this context, the collaboration between scientists and industry is crucially relevant.

Toby Maher (London, UK) presented an overview of the PROFILE study (NCT01110694 and NCT01134822), designed to answer the crucial questions of how we define IPF, predict disease course and stratify patients. The study also aims to identify new therapeutic targets and surrogate markers $[7,8]$. Recent studies identified several protein biomarkers in IPF; among others, CCL18 [9], circulating fibrocytes [10], matrix metalloprotease (MMP)-7, intercellular adhesion molecule-1, interleukin (IL)-8, vascular cell adhesion molecule-1 and S100A12 [11]. Furthermore, gene expression analysis has highlighted a number of key genes differentially expressed in IPF, compared with other fibrotic lung disorders or in slow as compared with rapid disease [12]. The PROFILE study will prospectively seek to validate these, and other, markers and will also assess longitudinal change in markers in relation to disease progression. More than 250 IPF patients have already been recruited and preliminary results indicate that telomere length may predict disease progression. Moreover, the innovative concept that microbes can contribute to disease course is being explored through the analysis of the so-called IPF lung "microbiome". Finally, home monitoring using portable spirometers seems to be a promising and cost-effective way of caring for IPF patients, improving both monitoring and detection of disease complications.

Shelia Violette (Cambridge, MA, USA) discussed the innovative concept of using biomarkers to monitor the effect of an anti-fibrotic drug in the context of a phase II trial. The challenges of drug development, in particular in the field of pulmonary fibrosis, have been illustrated in the context of a cultural problem: translational medicine could represent a unique option to reduce technical and clinical development risks, while reducing the costs of studies, currently set at the level of hundreds of millions of US dollars. The example of STX-100, a humanised monoclonal antibody to integrin $\alpha \mathrm{v} \beta 6$ to treat IPF, has been presented as an example of the attempt to link, in a phase II study, a biological hypothesis (i.e. $\alpha \mathrm{v} \beta 6$ blockade will attenuate transforming growth factor (TGF)- $\beta$ activity in the lung) with a clinical hypothesis (i.e. attenuation of TGF- $\beta$ activity in the lung will preserve lung function). Given the limitation of early phase II studies, the clinical hypothesis is virtually impossible to test and, therefore, the potential benefits of an approach based on rigorous quantification of the biological hypothesis have been discussed. An attractive solution to test this biological hypothesis has been presented, based on the monitoring of TGF- $\beta$ activity in bronchoalveolar lavage (BAL) cells from IPF patients: alveolar macrophages could, therefore, be considered as biosensors of $\alpha \mathrm{v} \beta 6$-mediated TGF- $\beta$ activation in the fibrotic lung. The data generated by a scientific collaboration between industry and a number of academic institutions support the feasibility of phase II trials with a sequential dose-escalation design and with safety, pharmacokinetics and impact on BAL biomarkers as major end-points. New clinical phenotypes are emerging from these studies and might identify in the future targets for innovative management strategies.

Ivan O. Rosas (Boston, MA, USA) discussed the challenges related to the identification of patients with subclinical interstitial lung disease, i.e. those individuals who have interstitial lung abnormalities (ILA), as identified by chest high-resolution computed tomography [13]. Based on the available epidemiological data, it can be hypothesised that in IPF, ILA could actually precede the onset of symptoms by more than 10 years. Even in the absence of symptoms, when compared to healthy controls patients with ILA have significantly lower diffusion capacity [14] and increased levels of tissue MMP7 [15]. Unpublished results from microarray experiments on lung tissue from controls, IPF and ILA patients suggest that gene expression profiles are similar in IPF and ILA, thus, supporting the concept of ILA as the initial step of IPF. Further analysis of participants enrolled in the COPDGene study [13] suggests that those affected by metabolic syndrome (i.e. body mass index $\geqslant 30 \mathrm{~kg} \cdot \mathrm{m}^{-2}$, diabetes and arterial hypertension) could be at higher risk of developing ILA. Recent findings suggest that select groups at risk of developing ILD have reduced lung volumes, increased respiratory symptoms and reduced exercise capacity, which has not been recognised by 
either the subject or a physician. Given the potential clinical impact, longitudinal studies are required to better define the natural history of subclinical ILD.

Vincent Cottin (Lyon, France) illustrated the clinically important and scientifically intriguing relationships between emphysema and pulmonary fibrosis. Although the combination of emphysema and fibrosis in the lung has been known for a long time [16], its impact on diagnostic [17] and prognostic [18] aspects is still a topic for clinical research. Recent data show that emphysema is common in IPF (20-50\% of cases) [19] and patients with combined pulmonary fibrosis and emphysema (CPFE) have distinct features, like very low diffusion capacity [20]. Vincent Cottin provided clinical data in support of the identification of distinct interrelationships between emphysema and fibrosis, ranging from being separate entities, to a progressive transition, to the combination of paraseptal emphysema with fibrosis, with or without thick-walled large cysts. In silico models of CPFE are being developed that may allow simulation of CPFE and prediction of its evolution. The fact that coexisting emphysema may delay the decrease in vital capacity [21] is critical for trial design and will need to be taken into account in future studies. To do so, semi-quantification of emphysema in CPFE is a field of active research [22]. Finally, CPFE has been shown to be a distinct entity not only in patients with IPF, but also in those with connective tissue disease [23] and occupational exposures [19]. Pathogenesis beyond smoking, definition criteria, impact of emphysema on progression of fibrosis, and optimal therapeutic options and management are all open issues for future clinical research.

\section{Invited oral presentations}

23 of the 104 accepted abstracts were selected for brief oral presentations and discussion. An attempt was made to include a mix of junior and more senior investigators. The role of the extracellular matrix in induction and maintenance of pulmonary fibrosis was the topic of a number of these presentations. A general theme, first introduced by Geoff Laurent in the 1980s [24] but recently supported by mechanistic data [25], was that the lung extracellular matrix is dynamic and constantly remodelling. Jack Gauldie (Hamilton, Canada) presented data suggesting that mechanical tension may induce activation of latent TGF- $\beta$ in the lung. The results of experiments in a tissue bath apparatus using a force transducer and increased ventilatory pressure on lung slices and fibrotic lungs indicate that TGF- $\beta$ activated in fibrotic tissue strips signals through the TGF- $\beta$ receptor in the tissue and activates nuclear translocation of phosphorylated SMAD2. Janette Burgess (Sydney, Australia) provided data supporting the concept of the extracellular matrix playing a bioactive role in lymphangioleiomyomatosis (LAM), by modulating the expression of key molecules, like fibronectin, periostin and fibulin-1, which are all increased in the tissue samples from LAM patients. The serum levels of fibulin-1 are significantly increased in LAM, compared with female nondiseased controls.

Several presenters also discussed the need to refine our current animal model of pulmonary fibrosis (mainly bleomycin) and improved readouts thereof (such as micro computed tomography (CT) and lung function measurements). It was recognised that the current model has limitations with regard to the human counterpart of IPF (e.g. it is primarily inflammatory and self-limited), but its merits as a convenient, welldescribed and mechanistically informative model were also recognised. Chris Scotton (London, UK) assessed the role of ex vivo micro-CT scans in evaluation of pulmonary fibrosis in the bleomycin rodent model. The data presented support the concept that ex vivo micro-CT can rival traditional end-point measures of fibrosis and is a highly sensitive and robust measure of lung fibrotic changes. Emilie Krafft (Liège, Belgium) presented data on a model of spontaneous canine IPF, generated in the context of the European LUPA project, which is aimed at unravelling common human diseases using dog genetics. By using microarray techniques and validation with quantitative PCR, about 800 differentially expressed canine genes were identified. In particular, and analogous to human data, genes related to leukocyte chemotaxis (e.g. CCL2, CCL17 and IL-8) were upregulated, while genes related to matrix remodelling (e.g. MMPs) were downregulated. These findings suggest a possible common pathway between the spontaneous human and canine disease.

The relationship of IPF to ageing was recognised and ageing was the focus of several presentations. Claude Jourdan Le Saux (San Antonio, TX, USA) showed that in the bleomycin mouse model of pulmonary fibrosis, a novel small molecule activator of telomerase (GRN510) could enhance the telomerase function in the lung with a cell-specific effect and reduces the extent of the fibrotic response. Joyce Lee (San Francisco, CA, USA) presented a cohort study on 59 IPF patients suggesting that shorter telomeres are associated with increased radiological fibrosis score and shorter survival. Mauricio Rojas (Pittsburgh, PA, USA) introduced the concept of the increased susceptibility of the aged lung to injuries. In a bleomycin-induced mouse model of lung fibrosis he showed that aged mice are more susceptible to herpes virus-induced lung injury, although aged mice control virus infection similar to young mice. Bethany Moore (Ann Arbor, MI, USA) discussed the role of periostin, an extracellular matrix protein, in relation to age and regulation of lung fibrosis. This molecule is elevated in the serum of IPF patients and in IPF fibroblasts and correlates with 
lung function decline. Fibroblasts from aged mice overproduce periostin and are protected from apoptosis, thus suggesting that this molecule may contribute to the mechanisms of age-related fibrosis.

Several mechanisms of pulmonary fibrosis were discussed in the context of the identification of potential therapeutic targets. Claire Calderwood (Southampton, UK) highlighted the role of histone deacetylase inhibitors as potent anti-fibrotic agents. Data on lung explant tissue, from IPF and healthy controls, and on in vitro cultured fibroblasts indicate that FK228 (romidepsin, a drug approved by FDA for use in cutaneous and peripheral T-cell lymphoma) suppresses fibroblast proliferation and differentiation, induces p21 expression, and suppresses lysyl oxidase (LOX) mRNA and protein expression. In light of the increased levels of LOX in the BAL fluid from IPF patients, increasing histone acetylation represents an attractive therapeutic strategy in IPF. Annie Pardo (Mexico City, Mexico) discussed MMPs in IPF. Increased MMP19 gene expression and protein levels in affected epithelial regions and BAL fluid have been identified: MMP19 is also overexpressed in the bleomycin-induced mouse model of pulmonary fibrosis. The concept that different genetic backgrounds for different MMP expression levels can lead to protection (MMP19) from or susceptibility (MMP7) to pulmonary fibrosis was discussed. Katerina Antoniou (Crete, Greece) discussed citrullination, a post-translational modification of arginine by peptidylarginine deiminase enzymes, as potential key factor in the pathogenesis of pulmonary fibrosis. Western blot analyses on 53 patients with IPF and 37 with progressive rheumatoid lung disease indicate that citrullination enzymes may predict late mortality. Jason Chien (Seattle, WA, USA) showed that elevated baseline serum lysyl oxidase 2 (LOXL2) levels are associated with higher risk for IPF progression. These data, derived from serum samples from a negative trial with ambrisentan, support the evaluation of a specific noncompetitive allosteric inhibitor of LOXL2 in an ongoing phase II clinical trial in IPF patients. Barry Shea (Boston, MA, USA) explored the hypothesis that increased vascular permeability after lung injury promotes fibrosis through intra-alveolar coagulation. Data generated in the bleomycin mouse model indicate that sphingosine-1-phosphatesphingosine-1-phosphate receptor 1 signalling promotes non-fibrotic resolution of lung injury by limiting intra-alveolar coagulation, thrombin-PAR1 signalling and TGF- $\beta$ activation. Interestingly, anticoagulation with warfarin does not attenuate fibrosis in a vascular leak-dependent model and this experimental observation supports the recent finding of no benefit to warfarin therapy in patients with IPF [26]. Amanda Tatler (Nottingham, UK) discussed the role of the $\alpha v \beta 6$ integrin in IPF. Smad 3 mediates TGF- $\beta$-induced $\alpha v \beta 6$ expression and the loss of Smad3 reduces TGF- $\beta$-induced upregulation of $\alpha \mathrm{v} \beta 6$. Some IPF patients have increased binding of Smad3 to the ITGB6 promoter, while others have decreased binding of the repressor Elk-1 to the ITGB6 promoter. Jeffrey Horowitz (Ann Arbor, MI, USA) reported new findings on the inhibition of apoptosis proteins to regulate IPF fibroblast resistance to Fas-mediated apoptosis. Although it has been shown that pro-fibrotic mediators induce fibroblast resistance to apoptosis [27], IPF is still characterised by the so-called "apoptosis paradox", in which increased epithelial cell apoptosis contrasts with decreased mesenchymal cell apoptosis. Recent ex vivo experiments indicate that inhibitors of apoptosis family proteins are increased in a subset IPF lung fibroblasts, are expressed within fibroblastic foci of IPF tissue, are induced in normal lung fibroblasts by pro-fibrotic mediators and are suppressed in normal lung fibroblasts by anti-fibrotic mediators. Finally, specific targeting of IAP (inhibitor of apoptosis protein) could sensitise IPF lung fibroblasts to apoptotic stimuli, thus representing a potential therapeutic strategy. Guoying Yu (Pittsburgh, PA, USA) showed that miR-29, a small, noncoding RNA, is downregulated in human IPF lungs and its mimicry could attenuate pulmonary fibrosis in the bleomycin mouse model, thus making the class of microRNAs new potential therapeutic targets for fibrotic lung disorders. Cecilia Sanchez (New Orleans, LA, USA) pointed to autophagy, an evolutionarily conserved catabolic mechanism involving cellular degradation of misfolded or long-lived proteins and damaged organelles, as a critical process in the development of fibrosis. She showed that during fibroblast-myofibroblast differentiation, TGF- $\beta 1$ can inhibit autophagy and that the induction of autophagy may block this differentiation process and increase collagen degradation. The potential of resveratrol as a molecule able to overcome the effects of TGF- $\beta 1$ on autophagy was also discussed. These data formed the basis for the provocative hypothesis that therapies directed toward maintaining organ function through longevity may reduce lung fibrogenesis. On the same line, Avignat Patel (Boston, MA, USA) showed that a marker of autophagy, LC3-II, is decreased, while p62 is accumulated, in the IPF lung [28]. TGF- $\beta 1$ can inhibit autophagy in lung fibroblasts by modulating TIGAR (a p53 induced glycolysis and apoptosis regulator). Interestingly, rapamycin (an mTOR inhibitor, which reduces fibronectin and $\alpha$-smooth muscle actin expression in the lung) protects against bleomycininduced fibrosis, potentially through autophagy induction.

The potential of regenerative medicine also emerged during some presentations. Luis Ortiz (Pittsburgh, PA, USA) introduced the concept that the mesenchymal stem cell "secretome" (i.e. the secreted microvesicles) could reprogramme innate immunity in environmentally induced lung diseases. Mesenchymal stem cell microvesicles (exosomes) might convey microRNAs targeting genes regulating the innate immune system that could impact on the outcome of pulmonary fibrosis. Using microRNA microarray and quantitative 
PCR techniques, it has been shown in silica-exposed mice that mesenchymal stem cell-derived exosomes are phagocytised by macrophages, inducing nuclear factor-kB activation and M1 polarisation. Exosomes reduce silica-induced fibrosis, reduce expression of fibrotic and inflammatory genes, and preserve the expression of epithelial genes. These data suggest that mesenchymal stem cell-derived exosomes might become a tool to modify the tissue pro-fibrotic pathways in the silicosis lung.

Joe Lasky (New Orleans, LA, USA) discussed the possibility of repositioning "old" drugs as anti-fibrotics, by using the example of nelfinavir, a drug approved in 1997 as a protease inhibitor to treat HIV infection. In vitro data show that nelfinavir inhibits fibroblast differentiation and collagen gel contraction, by blocking TGF- $\beta$ Smad $2 / 3$ phosphorylation. These findings provide further support to the evidence, from animal models, that the delayed administration of another proteasome inhibitor (bortezomib) blocks the development of pulmonary fibrosis [29]. Given the large amount of data on the safety and tolerability of drugs already used for other indications, the concept of transferring "old" drugs to new indications is attractive. Arata Azuma (Tokyo, Japan) presented an update on post-marketing surveillance of the antifibrotic drug pirfenidone in patients with IPF in Japan, where the drug has been approved in 2008. The data presented were on 973 patients enrolled in a post-marketing surveillance study, including all stages of disease severity. About one-third of patients discontinued pirfenidone due to drug-related adverse events, most often decreased appetite, photosensitivity reactions and nausea. Patients treated with prophylactic drugs for gastrointestinal side-effects reported significantly reduced gastrointestinal side-effects, as compared to those untreated. Data on per cent predicted vital capacity decline after 12 months of treatment in 263 patients indicate a mean decline of $1.9 \%$ (with a standard deviation of $14.7 \%$ ). Although limited and uncontrolled, these data provided useful information about real-world pirfenidone use in IPF.

New interesting observations emerged from clinical studies. Christopher Ryerson (Vancouver, Canada) presented the data from a large retrospective study, assessing the prevalence and the prognosis of patients with unclassifiable ILD. These patients represent a sizeable $(\sim 10 \%)$ fraction of all ILDs and, as such, are the fourth most common group. They usually cannot be classified due to patient unwillingness to perform surgical lung biopsy. Overall prognosis is intermediate between IPF and non-IPF ILD, with the risk of death or disease progression associated with fibrosis score and diffusion capacity. Since these patients are relatively common in clinical practice, there is a need to evaluate their optimal clinical and therapeutic management. Tamera Corte (Sydney, Australia) discussed the clinically relevant issue of pulmonary hypertension associated with idiopathic interstitial pneumonias. A clinical study showed that the relative increase in pulmonary blood flow during exercise is reduced in pulmonary hypertension patients compared to idiopathic interstitial pneumonia patients and normal controls. Moreover, resting and end of exercise serum levels of the brain natriuretic peptide are increased in pulmonary hypertension patients compared to idiopathic interstitial pneumonia patients, who have higher levels than normal controls. The noninvasive measurement of pulmonary blood flow during exercise may represent a useful method for identifying patients with pulmonary hypertension, or identifying small functional changes in exercise.

\section{Future directions in pulmonary fibrosis research}

A major aim of this ICLAF meeting was to explore the current challenges we face as a research community and identify where continued or new research focus is needed (fig. 2). To address this aim, participants were assigned to half-day breakout sessions. These sessions were facilitated by leading investigators in the field and encouraged interaction among basic scientists, clinical scientists, clinicians, industry colleagues and advocacy group members. All breakout sessions were asked to address two fundamental questions. First, why have so few novel therapies been developed despite gains in the science of fibrosis? Second, where do we see the greatest need as a community moving forward and what should we do about it? The synthesis of the responses from the breakout sessions, described below, provides a useful roadmap for future work in pulmonary fibrosis.

Why have so few novel therapies been developed despite gains in the science of fibrosis? A major challenge to the discovery of effective treatments for pulmonary fibrosis is the lack of insight into the prime target(s) relevant to human disease. The limitations of the available animal models of pulmonary fibrosis represent a major barrier [30-32]; we need to improve our understanding of the hierarchy of mechanisms involved in pulmonary fibrosis through patient-based research. The anticipated redundancy of pathogenic pathways involved in pulmonary fibrosis makes it less likely that single drug agents will make major impacts on the course of disease. Combination approaches that target multiple mechanisms may be more successful. Targeting what appear to be final common pathways of fibrosis [7], including coagulation cascade products, prostanoids and TGF- $\beta$ signalling, may prove fruitful and need to be explored further.

More sophisticated study design in early phase clinical trials, including cohort enrichment, is essential. We need to use and expand on existing clinical cohorts in academia and industry, and invest in more 


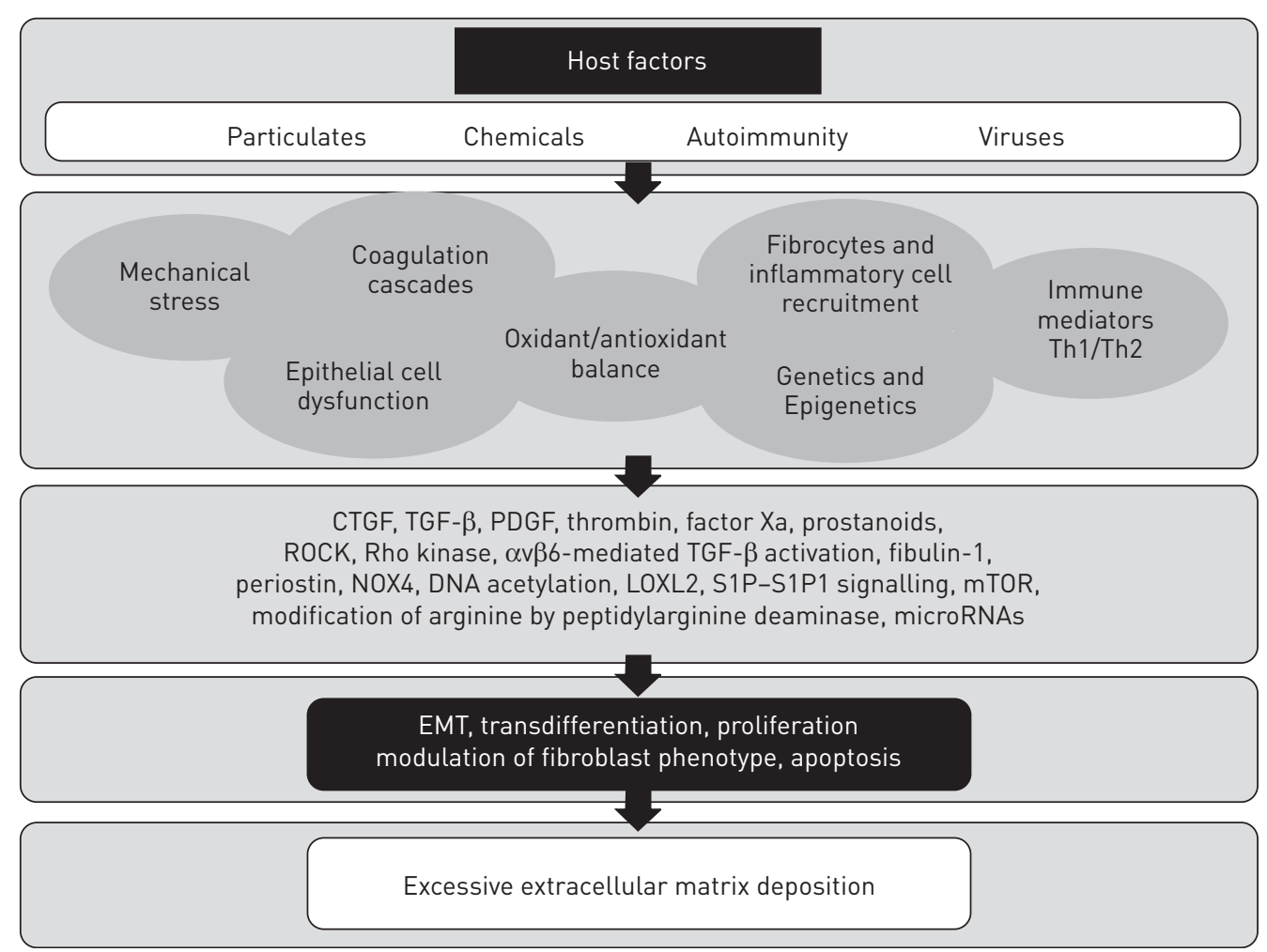

FIGURE 2 Schematic pathogenic steps in pulmonary fibrosis (as discussed during the 2012 ICLAF). Th: T-helper cell; CTGF: connective tissue growth factor; TGF: transforming growth factor; PDGF: platelet-derived growth factor; ROCK: Rho-associated protein kinase; NOX: NADPH oxidase; LOXL: lysyl oxidase; S1P-S1P1: sphingosine-1-phosphatesphingosine-1-phosphate receptor 1; mTOR: mammalian target of rapamycin; EMT: epithelial-mesenchymal transition.

sophisticated ones, to investigate and then validate potential subgroups of interest a priori, rather than rely on post hoc subgroup analysis. In all clinical studies, rigorous, detailed clinical data collection linked to biorepositories of blood and other tissues should be a high priority. Only the availability of large numbers of biological samples from well-defined cohorts of patients will allow for the rational and efficient identification of novel drug agents and the more sophisticated design of future clinical trials.

Efforts must be made to prioritise identification of target mechanisms in clinical trial design. Only through a better understanding the relevant mechanisms of disease will the chances of developing effective targeted therapies be realised. These therapies can then be tested in a rational, hierarchical clinical trial algorithm to assess combination therapy, an approach that has led to remarkable advances in oncology and HIV therapy in recent decades.

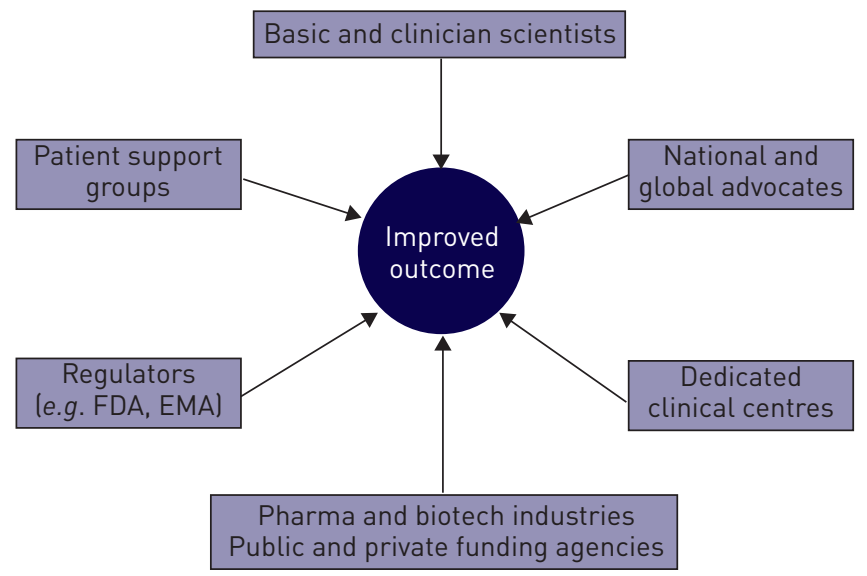

FIGURE 3 Collective efforts lead to improved outcomes for patients with pulmonary fibrosis. FDA: Food and Drug Administration; EMA: European Medicines Agency. 
To be successful, a close interaction between all stakeholders, clinicians, researchers, patients, industry colleagues, advocacy groups, regulators and other interested parties, will be necessary. Once we can show that the course of IPF is modifiable through pharmacological intervention, we believe the "flood gates will open" and we will see the development of numerous drugs for fibrotic lung disorders.

Where do we see the greatest need as a community moving forward and what should we do about it? There is a clear need for enhancing the integration across disciplines (basic science, translational, research, clinical research and clinical trials); such integration would catalyse the discovery process over the next decade. This extends to other disciplines as the mechanisms of fibrosis are almost certainly shared across organ systems. The involvement of people from hepatology, nephrology, dermatology, ophthalmology and other fields will be important.

The next generation of researchers and clinicians need to be encouraged and developed, and whenever possible should be actively involved at all levels of research and presentations. The importance of mentorship needs to be recognised and supported in the future. We must encourage a new generation of talented basic and clinician scientists to come into this field and create new ideas that will lead to improved therapeutic approaches.

Beyond the academic community, interested and invested partners need to be embraced. It is clear that to ensure research has relevance, all stakeholders, including patients, families, doctors, academics and basic researchers, need to be involved in the process of discovery. Each provides a valuable perspective on pulmonary fibrosis. Partnership with patients and advocacy associations will be particularly valuable; the achievements in the fields of lymphangioleiomatosis and cystic fibrosis demonstrate this clearly. It is absolutely clear that the likelihood of improving outcomes for patients with pulmonary fibrosis is dependent on collaborative and concerted efforts at every level of the discovery process.

\section{Closing comments}

The ICLAF meeting and other such international forums dedicated to integrative, collaborative and creative discussion are vital elements for scientific progress as we all work together to accomplish one common goal, to improve outcomes for patients with pulmonary fibrosis (fig. 3). The advances in basic and clinical science have been substantial since the first international colloquium on pulmonary fibrosis in 1980. At that time, our knowledge of the pathogenesis of fibrosis was rudimentary and the first placebo-controlled trial was many years off. While we should take some comfort in this progress over the past three decades, we are aware of the lack of sufficient progress towards therapy for patients with pulmonary fibrosis and we are motivated as a community to accelerate discovery.

The 18th ICLAF meeting will be held in Mont Tremblant, Quebec, Canada on September 20-24, 2014, after the annual European Respiratory Society conference (for more information and updates visit the website: www.iclaf.com). We look forward to meeting there and continuing the fight to improve the care of patients with pulmonary fibrosis.

\section{Acknowledgements}

The authors thank all participants and the sponsors of the 17th ICLAF and the E-Learning Centre of the University of Modena and Reggio Emilia, Modena, Italy (in particular Cinzia Tedeschi) for their excellent logistical and technical assistance during the meeting. Special thanks go to Stefania Cerri (University of Modena and Reggio Emilia, Modena, Italy) for her outstanding efforts in coordinating the event.

\section{References}

1 University of Modena and Reggio Emilia. The 17th International Colloquium on Lung and Airways Fibrosis, 2012. http://tv.unimore.it/index.php/29-canali-sp-356/registrazioni-video-sp-746/625-iclaf2012 Date last updated: January 10, 2013. Date last accessed: May 13, 2013.

2 Warburton D, Shi W, Xu B. TGF- $\beta$-Smad3 signaling in emphysema and pulmonary fibrosis: an epigenetic aberration of normal development? Am J Physiol Lung Cell Mol Physiol 2013; 304: L83-L85.

3 Huang X, Yang N, Fiore VF, et al. Matrix stiffness-induced myofibroblast differentiation is mediated by intrinsic mechanotransduction. Am J Respir Cell Mol Biol 2012; 47: 340-348.

4 Zhou Y, Huang X, Hecker L, et al. Inhibition of mechanosensitive signaling in myofibroblasts ameliorates experimental pulmonary fibrosis. J Clin Invest 2013; 123: 1096-1108.

5 Hecker L, Vittal R, Jones T, et al. NADPH oxidase-4 mediates myofibroblast activation and fibrogenic responses to lung injury. Nat Med 2009; 15: 1077-1081.

6 King TE Jr, Albera C, Bradford WZ, et al. Effect of interferon- $\gamma-1 \mathrm{~b}$ on survival in patients with idiopathic pulmonary fibrosis (INSPIRE): a multicentre, randomised, placebo-controlled trial. Lancet 2009; 374: 222-228.

7 Maher TM, Wells AU, Laurent GJ. Idiopathic pulmonary fibrosis: multiple causes and multiple mechanisms? Eur Respir J 2007; 30: 835-839.

8 Maher TM. Idiopathic pulmonary fibrosis: pathobiology of novel approaches to treatment. Clin Chest Med 2012; 33: 69-83. 
Prasse A, Probst C, Bargagli E, et al. Serum CC-chemokine ligand 18 concentration predicts outcome in idiopathic pulmonary fibrosis. Am J Respir Crit Care Med 2009; 179: 717-723.

10 Moeller A, Gilpin SE, Ask K, et al. Circulating fibrocytes are an indicator of poor prognosis in idiopathic pulmonary fibrosis. Am J Respir Crit Care Med 2009; 179: 588-594.

11 Richards TJ, Kaminski N, Gibson KF. Plasma proteins for risk prediction in idiopathic pulmonary fibrosis. Am J Respir Crit Care Med 2012; 185: 1329-1330.

12 Selman M, Pardo A, Barrera L, et al. Gene expression profiles distinguish idiopathic pulmonary fibrosis from hypersensitivity pneumonitis. Am J Respir Crit Care Med 2006; 173: 188-198.

13 Doyle TJ, Hunninghake GM, Rosas IO. Subclinical interstitial lung disease: why you should care. Am J Respir Crit Care Med 2012; 185: 1147-1153.

14 Rosas IO, Ren P, Avila NA, et al. Early interstitial lung disease in familial pulmonary fibrosis. Am J Respir Crit Care Med 2007; 176: 698-705.

15 Rosas IO, Richards TJ, Konishi K, et al. MMP1 and MMP7 as potential peripheral blood biomarkers in idiopathic pulmonary fibrosis. PLoS Med 2008; 5: e93.

16 Wiggins J, Strickland B, Turner-Warwick M. Combined cryptogenic fibrosing alveolitis and emphysema: the value of high resolution computed tomography in assessment. Respir Med 1990; 84: 365-369.

17 Wells AU, King AD, Rubens MB, et al. Lone cryptogenic fibrosing alveolitis: a functional-morphologic correlation based on extent of disease on thin-section computed tomography. Am J Respir Crit Care Med 1997; 155: 1367-1375.

18 Wells AU, Desai SR, Rubens MB, et al. Idiopathic pulmonary fibrosis: a composite physiologic index derived from disease extent observed by computed tomography. Am J Respir Crit Care Med 2003; 167: 962-969.

19 Jankowich MD, Rounds SI. Combined pulmonary fibrosis and emphysema syndrome: a review. Chest 2012; 141: 222-231.

20 Cottin V, Nunes H, Brillet PY, et al. Combined pulmonary fibrosis and emphysema: a distinct underrecognised entity. Eur Respir J 2005; 26: 586-593.

21 Akagi T, Matsumoto T, Harada T, et al. Coexistent emphysema delays the decrease of vital capacity in idiopathic pulmonary fibrosis. Respir Med 2009; 103: 1209-1215.

22 Schmidt SL, Nambiar AM, Tayob N, et al. Pulmonary function measures predict mortality differently in IPF versus combined pulmonary fibrosis and emphysema. Eur Respir J 2011; 38: 176-183.

23 Cottin V, Nunes H, Mouthon L, et al. Combined pulmonary fibrosis and emphysema syndrome in connective tissue disease. Arthritis Rheum 2011; 63: 295-304.

24 Laurent GJ. Dynamic state of collagen: pathways of collagen degradation in vivo and their possible role in regulation of collagen mass. Am J Physiol 1987; 252: C1-C9.

25 McKleroy W, Lee T-H, Atabai K. Always cleave up your mess: targeting collagen degradation to treat tissue fibrosis. Am J Physiol Lung Cell Mol Physiol 2013 [In press DOI:10.1152/ajplung.00418.2012].

26 Noth I, Anstrom KJ, Calvert SB, et al. A placebo-controlled randomized trial of warfarin in idiopathic pulmonary fibrosis. Am J Respir Crit Care Med 2012; 186: 88-95.

27 Horowitz JC, Ajayi IO, Kulasekaran P, et al. Survivin expression induced by endothelin-1 promotes myofibroblast resistance to apoptosis. Int J Biochem Cell Biol 2012; 44: 158-169.

28 Patel AS, Lin L, Geyer A, et al. Autophagy in idiopathic pulmonary fibrosis. PLoS One 2012; 7: e41394.

29 Mutlu GM, Budinger GR, Wu M, et al. Proteasomal inhibition after injury prevents fibrosis by modulating TGF- $\beta 1$ signalling. Thorax 2012; 67: 139-146.

30 Chua F, Gauldie J, Laurent GJ. Pulmonary fibrosis: searching for model answers. Am J Respir Cell Mol Biol 2005; 33: 9-13.

31 Moeller A, Ask K, Warburton D, et al. The bleomycin animal model: a useful tool to investigate treatment options for idiopathic pulmonary fibrosis? Int J Biochem Cell Biol 2008; 40: 362-382.

32 Seok J, Warren HS, Cuenca AG, et al. Genomic responses in mouse models poorly mimic human inflammatory diseases. Proc Natl Acad Sci USA 2013; 110: 3507-3512. 